\title{
HIFOMICETOS ACUATICOS DE SIERRA NEVADA Y SIERRA DE LOS FILABRES
}

\author{
Antonio ROLDAN, Enrique DESCALS y Mario HONRUBIA
}

\begin{abstract}
RESUMEN: Cincuenta y dos taxones de los denominados "hifomicteos acuáticos" ( 49 hifomicetos y anamorfos de asco-y basidiomicetos, y 3 zigomicetos) fueron detectados en muestras de espuma procedentes de rios y arroyos de Sierra Nevada. Se comenta la presencia de algunas especies y la aparición de varias formas desconocidas.
\end{abstract}

SUMMARY: "Acuatic Hyphomycetes" from Sierra Nevada and Sierra de los Filabres (South Spain). Conidia of fifty-two taxa of "aquatic Hyphomycetes" ( 49 aquatic Hyphomycetes and anamorphs of Asco- and Basidiomycetes, and 3 Zygomycetes) were found in foam samples from rivers and streams of Sierra Nevada and Sierra de los Filabres (S-Spain). The results are discussed and some unnamed forms are listed and drawn.

Palabras clave: Hifomicetos, Sierra Nevada, Sierra de los Filabres.

Key words: Hyphomycetes, Sierra Nevada, Sierra de los Filabres.

\section{INTRODUCCION}

En los cursos de agua que mantienen una vegetación de ribera bien conservada, la cantidad de material alóctono - procedente de ésta puede ser notable, sobre todo en los meses de otoño, coincidiendo con la caida de la hoja. Este material sufre diferentes procesos de descomposición y fragmentación en los que intervienen organismos de muy diversa índole.

El papel de los hongos en la descomposición de la materia vegetal es de gran importancia. La actividad fúngica es la más significativa durante las primeras etapas de este proceso (Bärlocher \& Kendrick, 1974), mientras que la acción bacteriana se incrementa posteriormente, una vez que la superficie colonizable va aumentando por efecto de la acción de hongos e invertebrados (Suberkropp \& Klug, 1976).

Muchos de estos hongos,que viven como saprófitos sobre materia vegetal sumergida, poseen conidios con formas estaurospóricas y escolecospóricas, presumiblemente adaptados al anclaje en medios lóticos. Estas formas no son exclusivamente fúngicas, ya que aparecen en otros grupos, como 
musgos y algas (Ingold, 1973). No obstante, debido a 1a presencia de tales conidios en dichos ambientes, constituyen un grupo ecológico que fue inicialmente denominado hifomicetos acuáticos por Ingold (1942). Este término no es rigurosamente exacto, ya que no todos los organismos en él incluidos son hifomicetos; y el carácter estrictamente acuático de muchos de ellos está por demostrar.

Descals et al. (1977) proponen el término "hongos ingoldianos", pero en la presente contribución se ha preferido conservar el nombre original por tratarse del más reconocido.

El estudio de los "hifomicetos acuáticos" se encuentra muy extendido por todo el mundo (ver bibliografía en Webster \& Descals, 1981). Sin embargo, en España se encuentra en sus comienzos. Varias zonas de la Peninsula, potencialmente ricas en esta particular micoflora, nunca han sido prospectadas. En el presente artículo se realiza un estudio de los "hifomicetos acuáticos", detectados en diversas campañas micológicas, procedentes de ríos y arroyos de Sierra Nevada y Sierra de los Filabres.

\section{MATERIAL Y METODOS}

La técnica utilizada para el estudio de estos hongos ha sido la observación directa de muestras de espuma.

En saltos de agua es frecuente ver acúmulos de espuma, que son particularmente abundantes después de una intensa 11uvia. Las muestras se recolectan en pequeños recipientes herméticos y se fijan con una mezcla de formaldehido, ácido acético y alcohol ( $1: 1: 5)$.

Para su examen, se secaron al aire unas gotas de espuma licuada sobre un portaobjetos y se tiñeron con lactofucsina.

En la Tabla 1 se expone un listado de localización y descripción de los cursos de agua muestreados.

\section{RESULTADOS}

En 1a Tabla 2 se relacionan las especies identificadas, en orden alfabético. Para cada taxon se indican las muestras en que se han detectado, con el número de orden que corresponde a la Tabla 1 .

\section{DISCUSION}

La flora fúngica de la zona prospectada guarda una cierta similitud con $1 \mathrm{a}$ de enclaves silíceos de Inglaterra (Ingold, 1975a), País Vasco (Descals et al., 1977), Albacete (Roldán et al., en prensa). Parece ser en consecuencia que, en climas templados con aguas ácidas, la distribución de los "hifomicetos acuáticos" sigue unas pautas generales. Esta teoría es mantenida por diversos autores (Webster \& Descals, 1981).

Las especies más abundantes son aquellas que poseen conidios tetrarradiados o ramificados. Articulospora atra (Fig. 1 D-G) presenta pequeños conidios con un corto eje basal, del que parten 3 brazos apicales. Se puede diferenciar de 


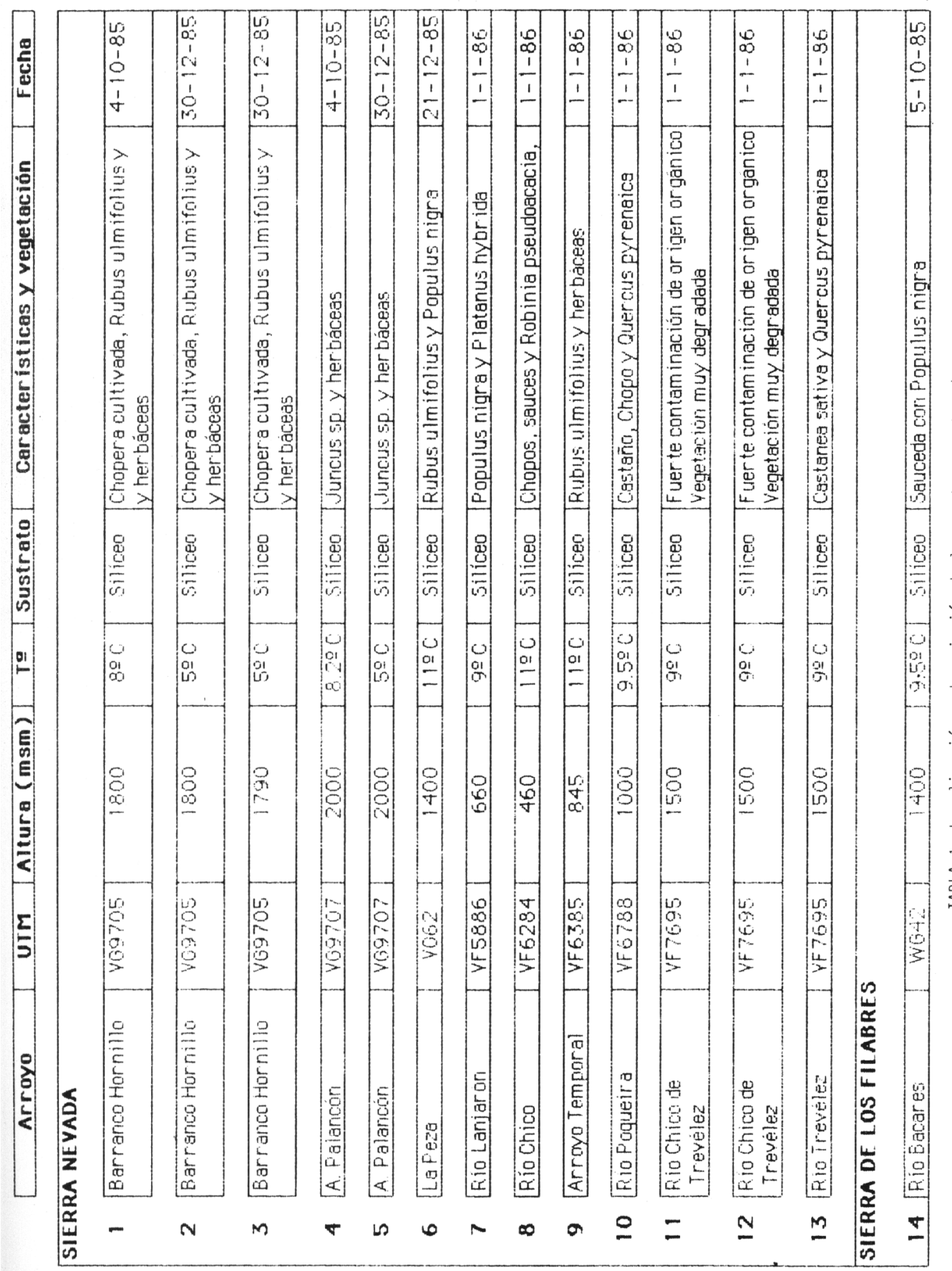


TABLA 2. RELACION DE TAXONES IDENTIFICADOS

1. Alatospora acuminata Ingold: $1,2,3,4,5,6,7,8,9,10,12,13,14$.

2. Alatospora flägellata (Gönczöl) Marvanová: 4 .

3. Alatospora pulchella Marvanová: $1,2,5,6,8,10,13$.

4. Anguillospora crassa Ingold: 1,2,3,4,5,9,10,13.

5. Anguillospora furtiva sp. inéd. Descals: 4 .

6. Anguillospora longissima (Sacr. E Sydow) Ingold: 4,6,7,8,12,14.

7. Anguillospora rosea sp. inéd. Descals: $1,2,4,6,13$.

8. Articulospora atra Descals: 2,3.

9. Articulospora tetracladia Ingold: $1,2,3,4,5,7,10,11,12,13$.

10. Camposporium pellucidum (Grove) Hugahes: 9.

11. Chaetospermum chaetosporum (Pat.) Smith \& Ramsbottom: 6,14.

12. Clavariopsis aquatica de Wild.: 5,9,10,13.

13. Clavatospora longibrachiata (Ingold.) Marvanová \& Nilsson: 1, 3,4,5,10,11,12,13.

14. Dendrospora erecta Ingold: $1,3,5$.

15. Dendrospora polymorpha Roldán E Descals: 6,14 .

16. Dendrospora tenella Delcals \& Webster: 1,2,5.

17. Diplocladiella scalaroides Arnaud: 6,9.

18. Dwayaangam cornuta Descals: 1,9 .

19. Entomophthora cf. thaxteri: 1 .

20. Erynia conica (Nowakowski) Remaudière \& Hennebert: $1,4,10,13$.

21. Erynia rhizospora (Thaxter) Remaudière E Hennebert: 4.

22. Flagellospora curvula Ingold: 1,2,3,7,10,11.

23. Gyoerffyella gemellipara Marvanová: 9.

24. Heliscella stellata (Ingold \& Cox) Marvanová: 1,2,3,7,10,13.

25. Heliscina campanulata Marvanová: 4 .

26. Heliscus lugdunensis Sacc. E Thérry: $1,2,4,5,6,8,9,10,14$.

27. Isthmolongispora sp.: 4 .

28. Lateriramulosa uniinflata Matsushima: 9.

29. Lemonniera aquatica de Wild.: 4,6.

30. Lemonniera cornuta Ranzoni: $3,5,8,9,10$.

31. Lemonniera pseudofloscula Dyko: 6 .

32. Lemonniera terrestris Tubaki: $1,2,3,5,7,8,9,10,12,13$.

33. Leptocladia neglecta Marvanová \& Descals: $9,10,13,14$.

34. Lunuspora curvula Ingold: 7,8,10,14.

35. Margaritispora aquatica Ingold:1,2,4,7,10,13.

36. Mycocentrospora acerina (Hartig) Deighton: $1,4,5,6,13$.

37. Taeniospora gracilis Marvanová: 3,4,5,13.

38. Tetrachaetum elegans Ingold: 7,10,13.

39. Tetracladium apiense Sinclair E Eicker: 7,10,13,14.

40. Tetracladium furcatum Descals: 10.

41. Tetracladium marchalianum de Wild.: $6,7,8,9,10,12,13,14$.

42. Tetracladium setigerum (Grove) Ingold: $1,4,5,6,7,9,10,13,14$.

43. Tricellula aquatica Webster: 2,9,10,13.

44. Tricellula graminea Ingold, Mc Dougall \& Dann.: 1,4,5,13.

45. Tricladium angulatum Ingold: 8,9,10,14.

46. Tricladium giganteum Iqbal: 1,4,5.

47. Tricladium patulum Marvanová \& Marvan: 13.

48. Tricladium robustum Marvanová: 1.

49. Tricladium splendens Ingold: 1,2,3,4,5,9,10,13.

50. Triposporina sp.: 8 .

51. ¿Varicosporium delicatum Iqbal: 1,4.

52. Volucrispora ornithomorpha (Trotter) Haskins:13. 
Alatospora, pulchella (Fig. 1 A) en que ésta última tiene dos brazos laterales. Articulospora tetracladia (Fig. 1 B-C) tiene una estructura parecida a A. atra, pero de mayor tamaño; y en ocasiones presenta ramificación secundaria (Fig. $1 \mathrm{C}$ ).

\section{Clavariopsis aquatica (Fig. 1 H-I) y Clavatospora}

longibrachiata (Fig. $1 \mathrm{~K}-\mathrm{M}$ ) constituyen una variante del diseño tetrarradiado, en que uno de los ejes es considerablemente más grueso que el resto.

El conidio ilustrado en $1 \mathrm{a}$ Fig. $1 \mathrm{~J}$ podría pertenecer a una especie de Entomophtora. Waterhouse \& Brady (1982) publican una clave para identificar las especies de este género, a partir de conidios, pero hay varias especies próximas que presentan morfología conidial parecida. No obstante, se trata de un propágulo trianseúnte. Otros hongos parásitos de insectos producen estauroconidios en medio acuático, como Erynia conica (Fig. 1 N-P) y Erynia rhizospora. Descals et al. (1981) mencionan la producción de propágulos ramificados en E. conica. A menudo, propágulos de este tipo aparecen ilustrados en la literatura, pero erróneamente identificados como rizópodos enquistados (Ingold, 1975b). Al igual que 1a anterior, E. rhizospora (Fig. 1 Q) también produce propágulos ramificados cuando germinan en inmersión. En la ilustración aparece un conidio primario que está dando lugar a un conidio secundario esférico. Posteriormente, éste desarrollará cuatro proyecciones divergentes, que son tubos germinativos.

Los conidios ramificados suelen ser de gran tamaño. En 1a figura 2 ( B, C y E) se ilustran varias especies de Dendrospora. otras formas no se adaptan a un diseño general y son estaurospóricas en sentido amplio (Fig. 3 G-I y L-T). A veces se insinúa el diseño tetrarradiado, como en Volucrispora ornithomorpha (Fig. 3 F), Heliscina campanulata (Fig. 3 G-I) y Heliscella stellata (Fig. 3 L-P). Lateriramulosa uniinflata (Fig. 3 T) tiene una morfología muy particular, que no sigue una pauta convencional.

El género Tricladium es uno de los mejor representados en la zona. T. robustum (Fig. 4 A y B) es una especie caracterizada porque el eje principal del conidio suele tener menos grosor que los brazos laterales (Marvanová, 1984). T. patulum (Fig. 4 E-F) presenta una ligera geniculación en el eje principal (Descals \& Webster, 1982).

Los conidios de Taeniospora gracilis (Fig. 4 G-F) guardan similitud con Tricladium, pero la presencia de una fíbula en el centro del eje principal delata su condición de anamorfo de un basidiomiceto. Nawawi et al. (1977) establecen 1a relación de este hongo con su teleomorfo Leptosporomyces galzinii (Bourd.) Jiilich.

Otro modelo morfológico, muy extendido entre "Hifomicetos acuáticos", es el conidio sigmoideo. Las especies que presentan estos conidios son muy conflictivas para su reconocimiento en espuma. En ocasiones, como en la figura $5 \mathrm{~F}$, que corresponde a Flagellospora curvula, parte del conidióforo se desprende y queda atrapado en la espuma, facilitando su identificación. Mycocentrospora acerina (Fig. 5 B y E) se reconoce por su apéndice basal, que puede ser septado (Fig. 5 E). Anguillospora rosea (Fig. 5 C) presenta conidios muy curvados.

En la Fig. 6 se han dibujado una serie de propágulos pertenecientes a hongos que creemos no descritos. Sin detalles sobre conidiogénesis y ontogenia, ya sea en cultivo puro o sobre el sustrato natural, no se pueden realizar descripciones de nuevas especies pero, si con posterioridad son descritas, la 


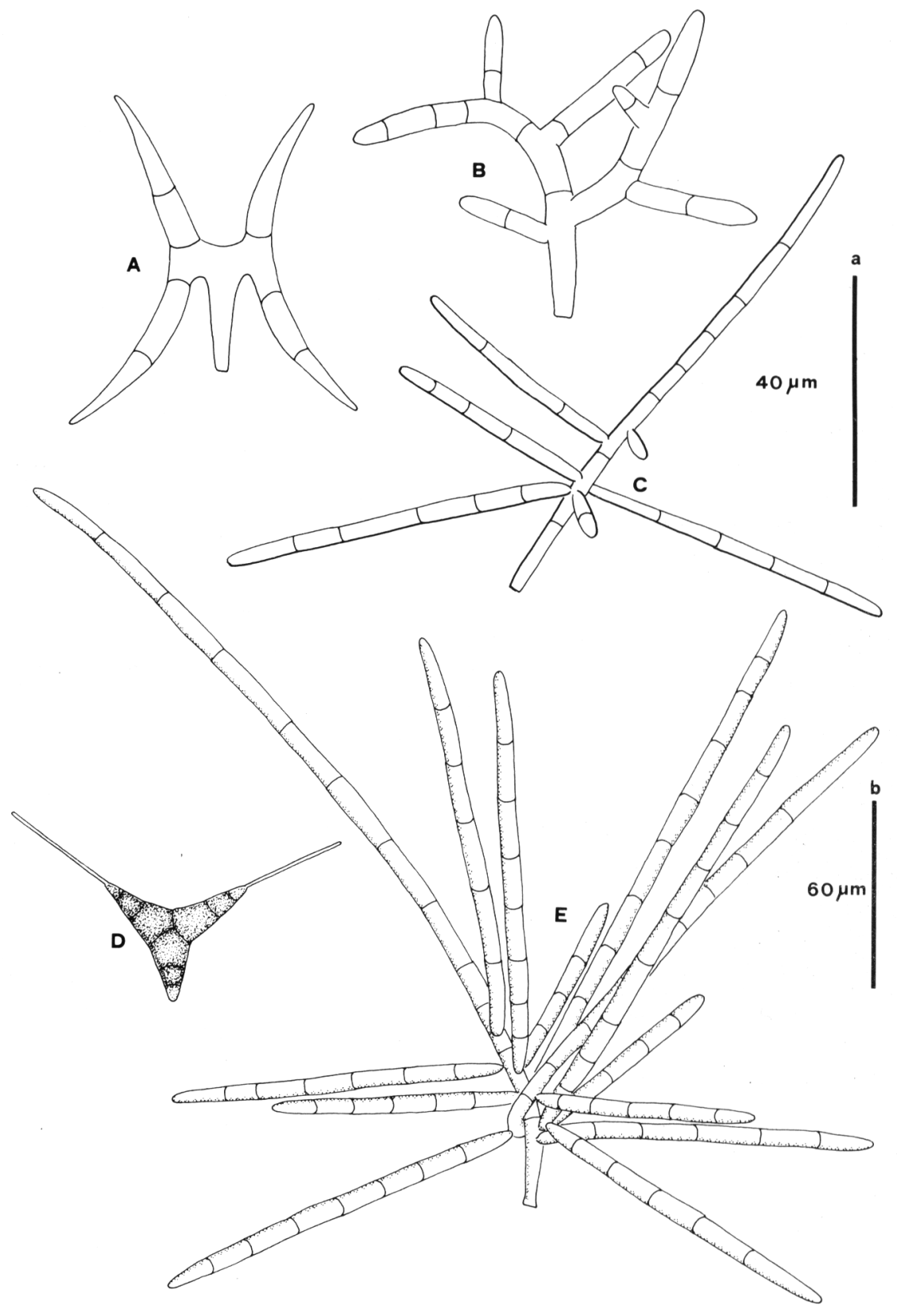

Figura 1 A: Alatospora pulchella, B-C: Articulospora tetracladia, D-G: A. atra, H-I: Clavariopsis aquatica, J: Entomophthora cf. thaxteri, K-M: Clavatospora longibrathiata, N-P: Erynia conica, Q: E. rhizospora. 

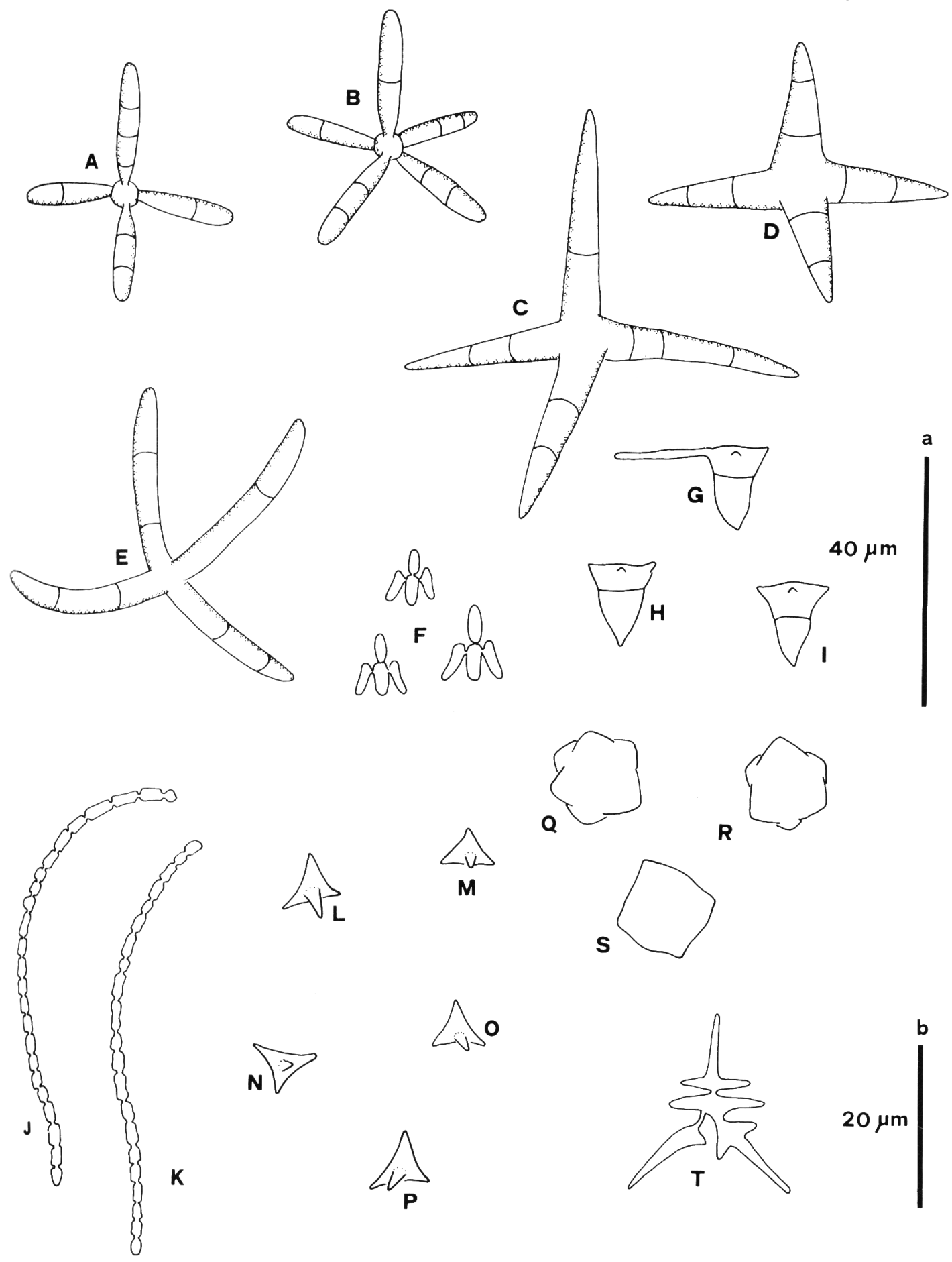

$40 \mu \mathrm{m}$
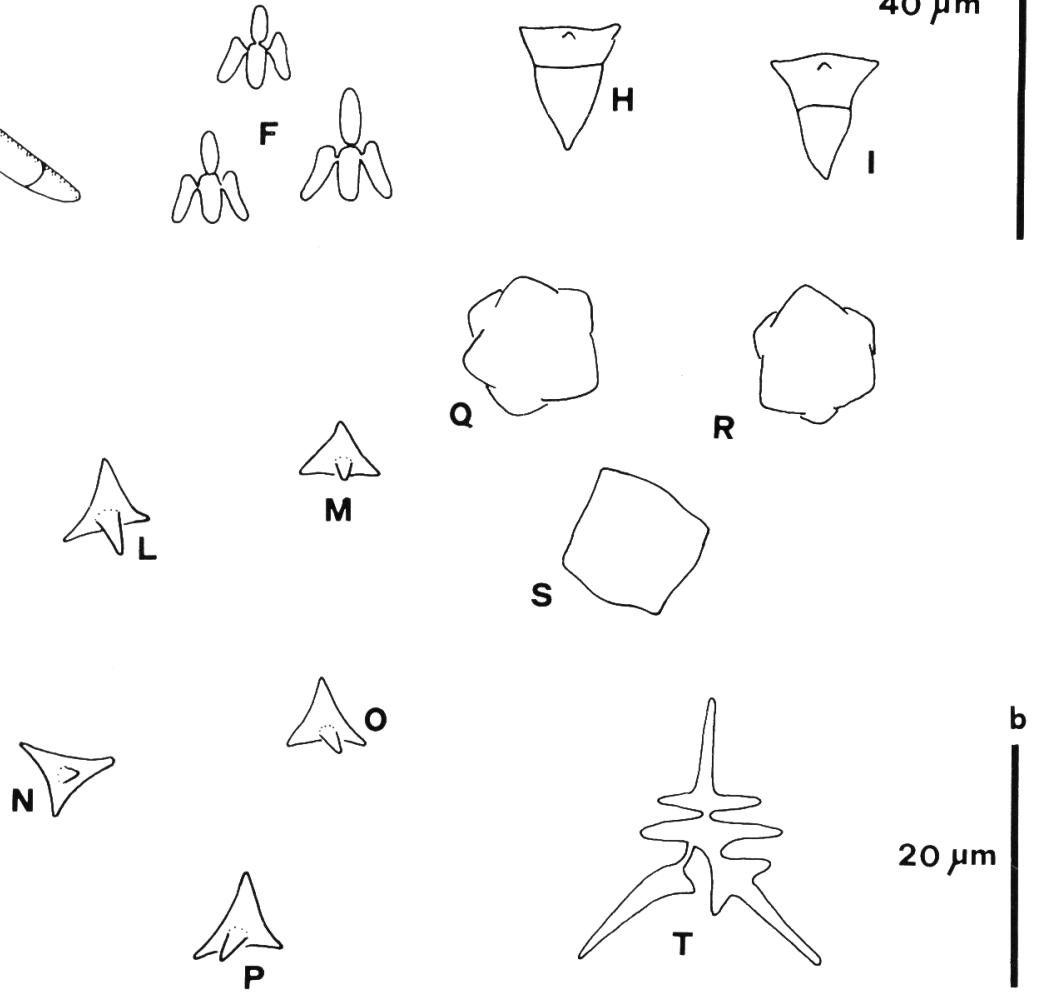

Figura 2 A: Dwayaangam cornuta, B: Dendrospora polymorpha, C: D. tenella, D: Diplocladiella scalaroides, $E$ : Dendrospora erecta ( $E$ = escala "b", resto = escala "a"). 


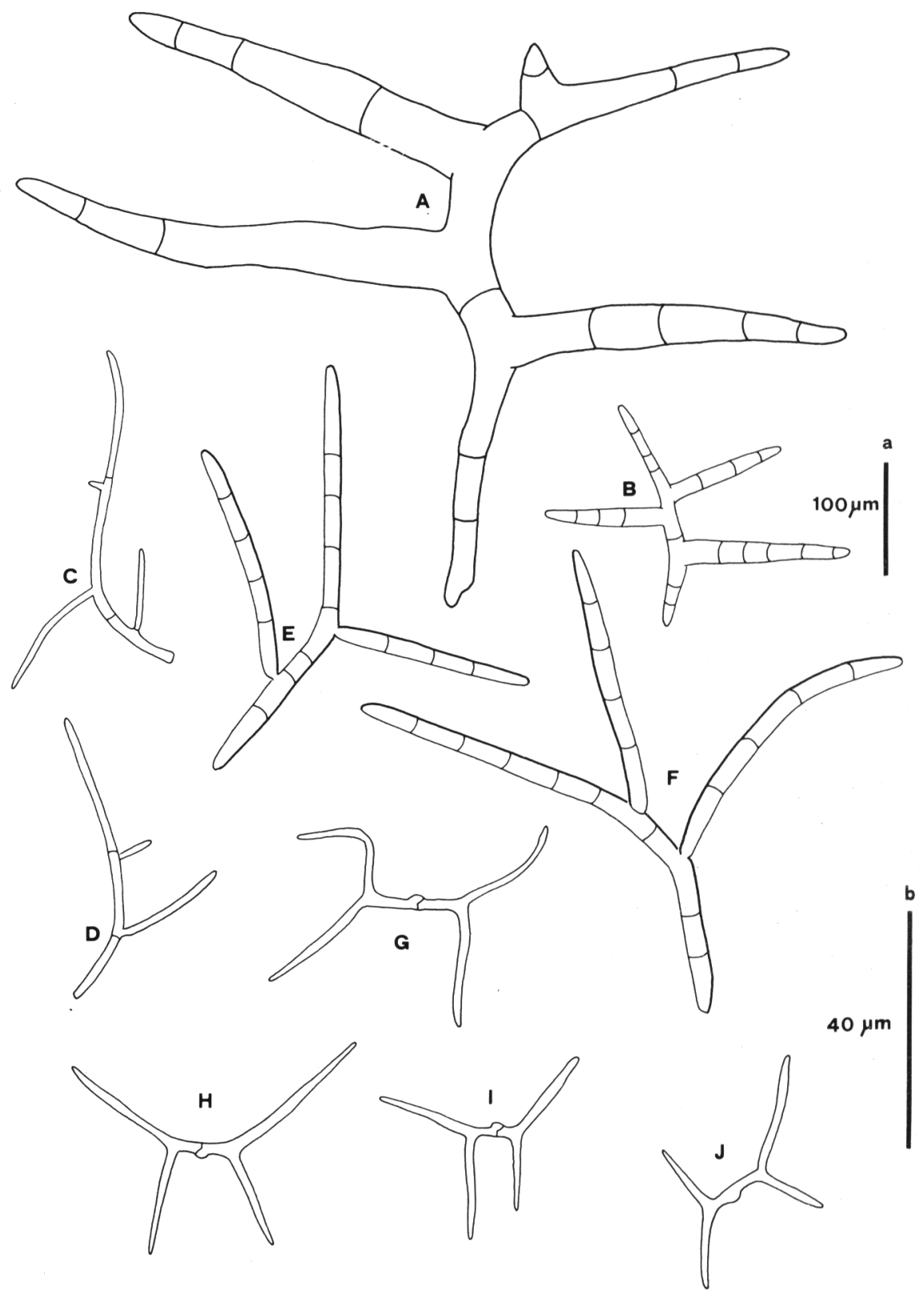

Figura 3 A-B: Lemonniera pseudofloscula, C-D: L. terrestris, E: L. cornuta, F: Volucrispora ornithomorpha, G-I: Heliscina campanulata, J-K: Isthmolongispora sp., L-P: Heliscella stellata, Q-S: Margaritispora aquatica, T: Lateriramulosa uniinflata ( $\mathrm{T}$ = escala "a", resto $=$ escala "b") 


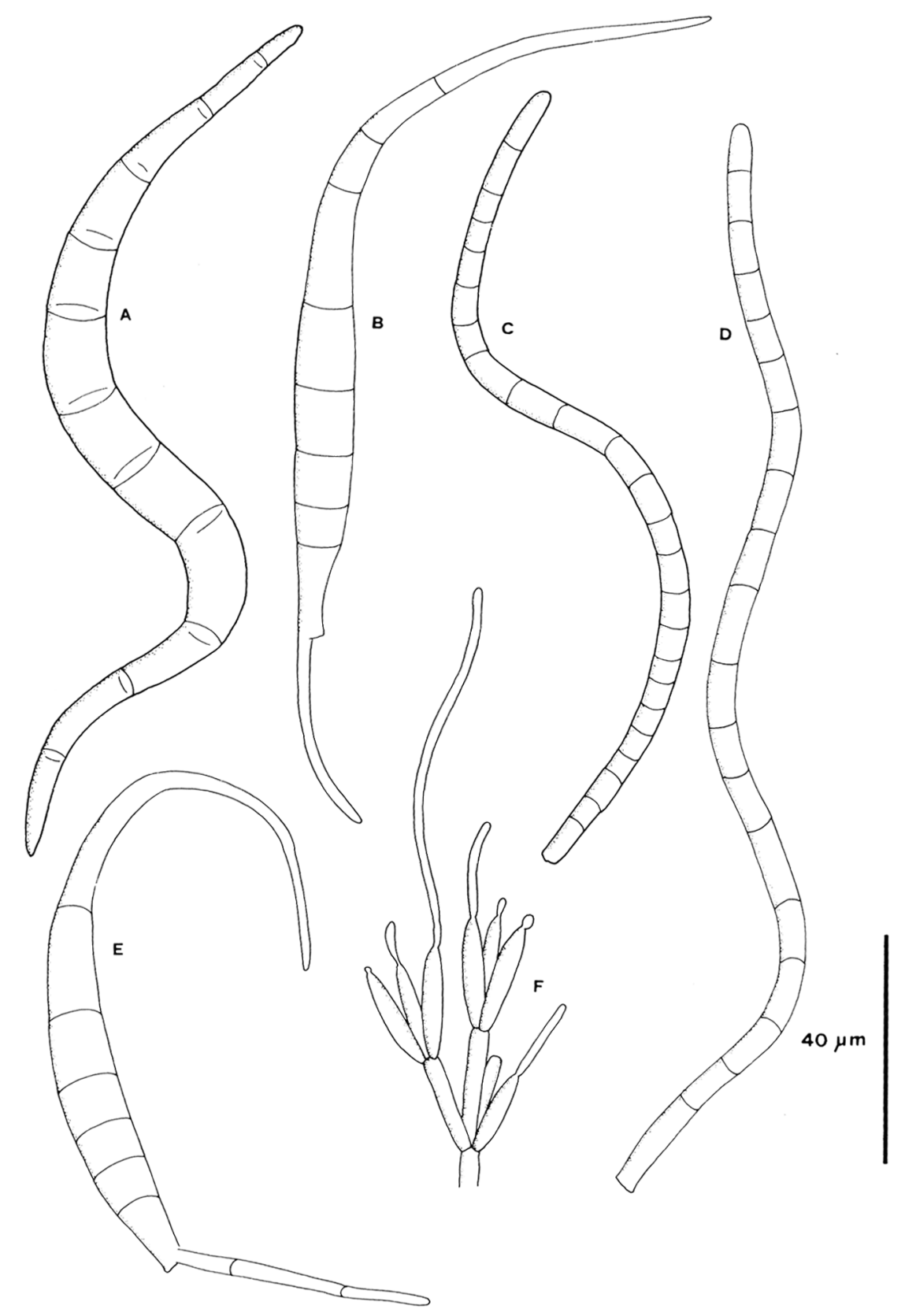

Figura 4 A-B: Iricladium robustum, C-D: ? Varicosporium delicatum, E-F: Iricladium patulum, G-I: Jaeniospora gracilis (B = escala "a", resto = escala "b"). 

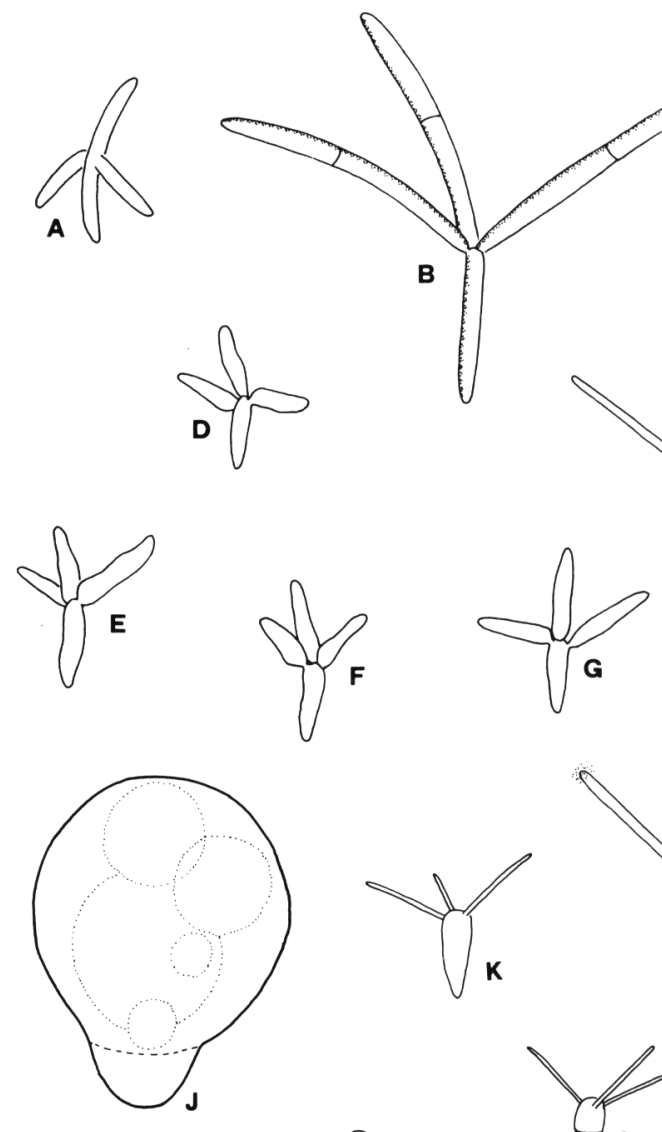

B
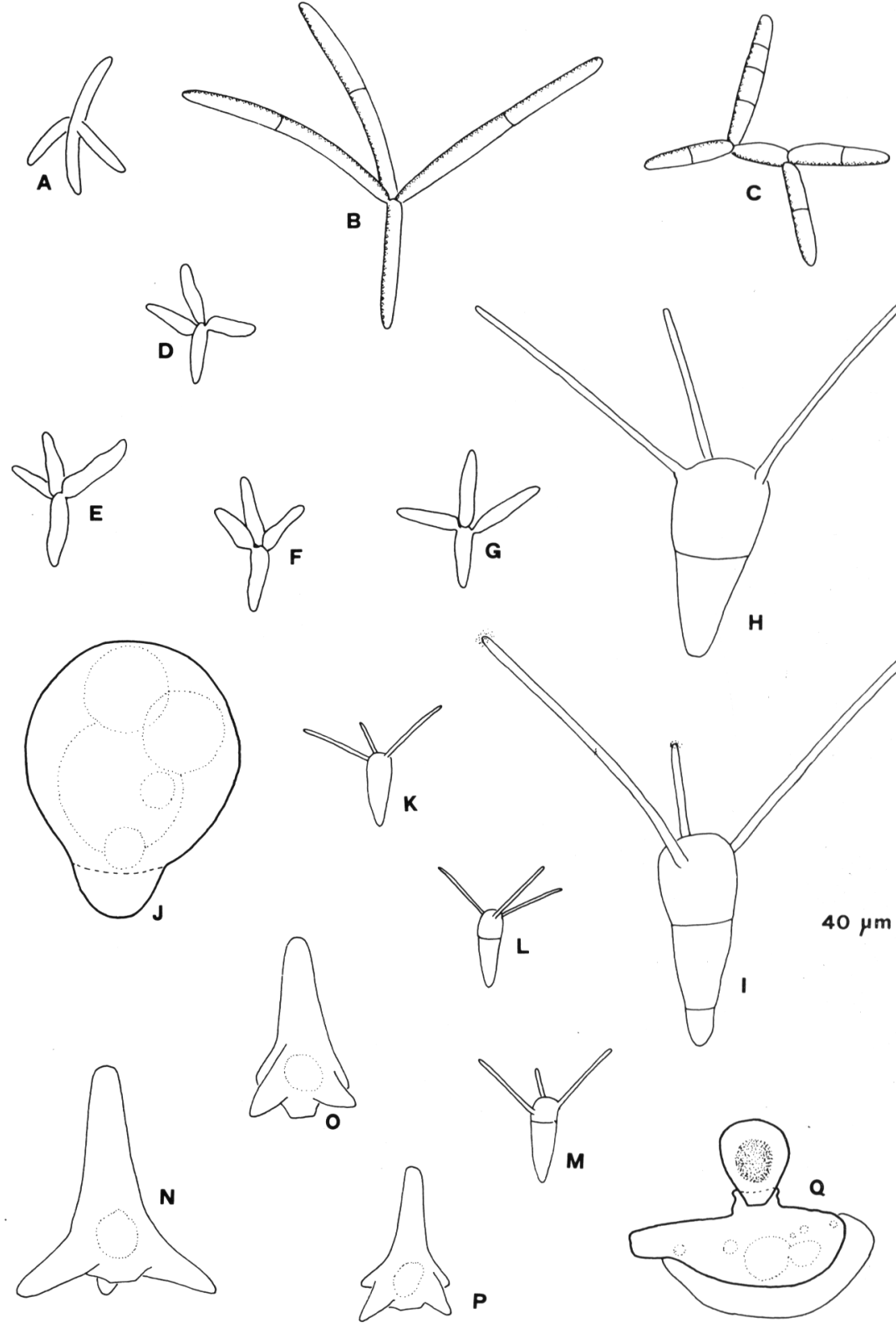

Figura 5 A: Anguillospora crassa, B-E: Mycocentrospora acerina, C-D: Anguillospora rosea, F: Flagellospora curvula. 


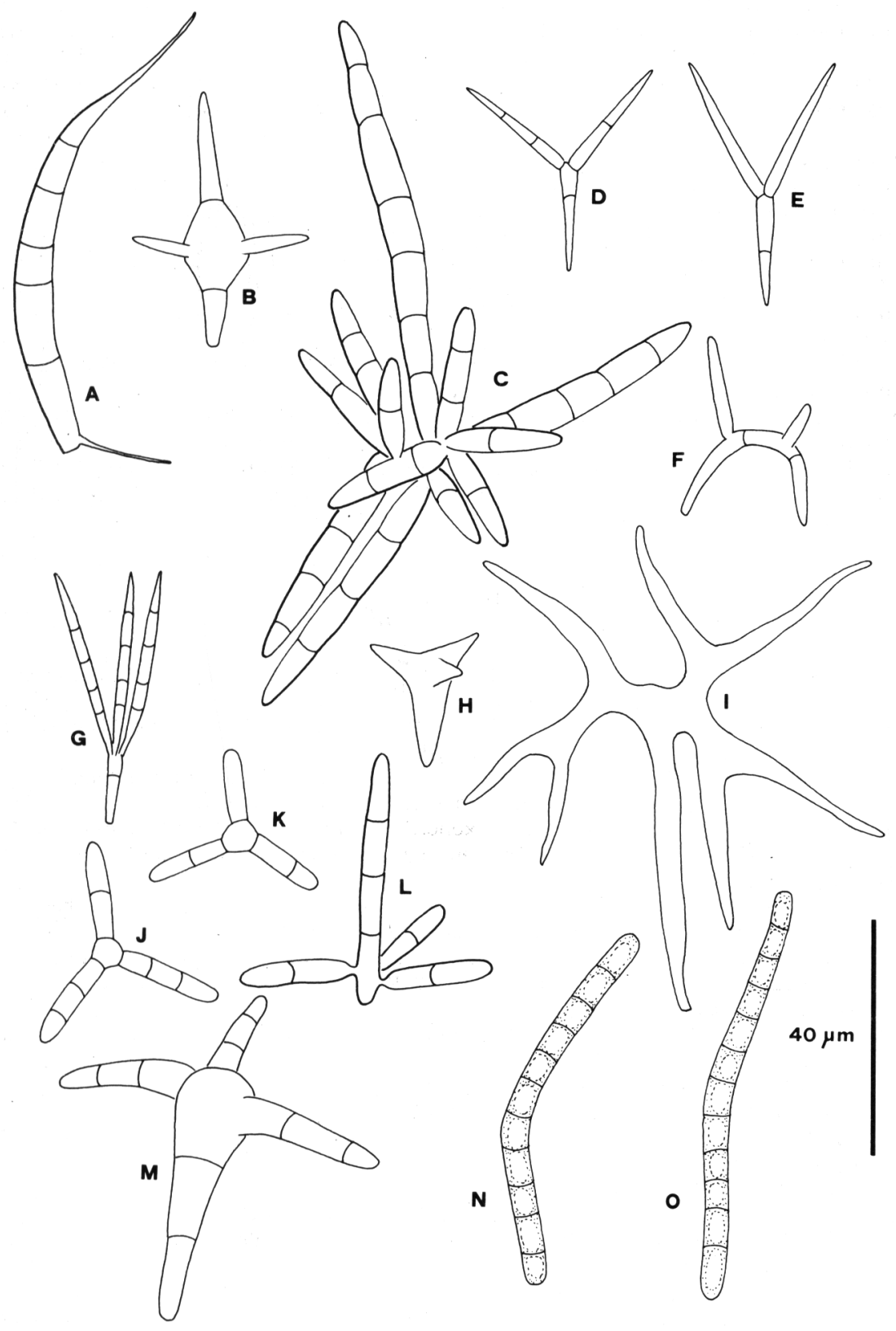

Figura 6: Formas desconocidas, A, D,E,H,J,K,I,L,M,N,0, (localidad 14), B,C (localidad 13). G, F (localidad 3). I (localidad 11). 
existencia de dibujos reconocibles en la literatura aporta datos valiosos sobre su fenologia y corología.

En la figura 6 A, la presencia de un apéndice en el punto de inserción del conidio lo acerca al género Mycocentrospora, pero también son varios los celomicetos que presentan conidios con esta morfología. La figura $6 \mathrm{C}$ nos muestra un conidio muy ramificado, atribuible al complejo Dendrospora-Dendrosporomyces. La especie más próxima es Dendrospora torulosa (Descals \& Webster, 1980), aunque ésta presenta el eje principal ligeramente curvado y en el caso de la figura es recto. La morfología del conidio 6 G lo hace asignable a los géneros Isthmotricladia o Tridentaria. La figura 6 H guarda semejanza con Heliscella stellata; aunque en este caso el tamaño es considerablemente mayor. Formas del tipo de la figura 6 I aparecen con frecuencia en muestras de espuma. Ya fueron ilustradas por Ingold (1975b). Por la ausencia de septación quizá se trata de un propágulo de algún zigomiceto.

E1 eje principal curvado y los brazos ligeramente constrictos en 1 a inserción, acercan el conidio 6 F a Tricladium curvisporum (Descals \& Webster, 1983), aunque las medidas no coinciden. La figura 6 L podría corresponder a un conidio del género Dendrospora o Triscelophorus. El resto de los propágulos ilustrados no admiten comparación con géneros conocidos.

\section{BIBL IOGRAF IA}

BARLOCHER, F \& KENDRICK, B -1974- Dynamics of the fungal population on leaves in a stream. Journal of Ecology 62:761-791.

DESCALS, E., SANDERS, P.F. \& UGALDE, U.0. -1977- Hifomicetos ingoldianos del País Vasco. Munibe 29:237-260.

DESCALS, E \& WEBSTER, J. -1980- Taxonomic studies on aquatic hyphomicetes. II. The Dendrospora aggregate. Trans. Br. mycol. Soc. 74:135-158.

DESCALS, E. \& WEBSTER, J. -1982- Taxonomic studies on aquatic hyphomycetes. III. Some new species and a new combination. Trans. Br. mycol. Soc. 78:405-437.

DESCALS, E \& WEBSTER, J. -1983- Four new staurosporous hyphomicetes from mountain streams. Trans. Br. mycol. Soc. 80:67-75.

DESCALS, E., WEBSTER, J., LADLE, M. \& BASS, J.A.B. -1981-Variations in asexual reproduction in species of Entomophthora on aquatic insects. Trans. Br. mycol. Soc. 77:85-102.

INGOLD, C.T. -1942- Aquatic hyphomicetes of decaying alder leaves. Trans. Br. mycol. Soc. 25:339-417.

INGOLD, C.T. -1973- Branched aquatic propagules with four $\circ$ five divergent arms. Acta Botánica Indica, 1:69-72.

INGOLD, C.T. -1975a- Conidia in the foam of two English stream. Trans. Br. mycol. Soc. 65:522-527.

INGOLD, C.T. -1975b- Hooker Lecture 1974. Convergent evolution in aquatic fungi: the tetraradiate spore. Biol. Jour. Linn. Soc. 7:1-25.

MARVANOVA, L. -1984- Two new Tricladium species from mountain streams. Mycotaxon 19:93-100.

NAWAWI, A., WEBSTER, J. \& DAVEY, R.A. -1977-Dendrosporomyces prolifer gen. et sp. nov., a basidiomycete with branched conidia. Trans. Br. mycol. Soc. 68:59-63.

ROLDAN, A., DESCALS, E. \& HONRUBIA, M. - Hifomicetos acuáticos en las cuencas altas de los ríos Segura y Guadalquivir. Anal. Biol. Univ. Murcia (en prensa) 
SUBERKROPP, K. \& KLUG, M.J. -1976- Fungi and baceria associated with leaves during processing in a woodland stream. Ecology 57:707-719.

WATERHOUSE, G. \& BRADY, B.L. -1982- Key to the species of Entomophthora sensu lato. Bull. Br. mycol. Soc. 16:113-143.

WEBSTER, J. \& DESCALS, E. -1981- Morphology, distribution and ecology of conidial fungi in freshwater habitats. In: Cole, G.T. and Kendrick, B. (Eds.) The Biology of Conidial Fungi. Academic Press. N.Y.:295-355. 